\title{
Radiofrequency ablation for intraductal extension of ampullary adenomatous lesions: proposal for a standardized protocol
}

\section{다)(1) $(5)$}

\author{
Authors \\ Andrea Tringali ${ }^{1,2}$, Maria Valeria Matteo ${ }^{1}$, Beatrice Orlandini ${ }^{1}$, Federico Barbaro ${ }^{1}$, Vincenzo Perri ${ }^{1,2}$, Qianqian Zhang $^{3}$, \\ Riccardo Ricci ${ }^{3,4}$, Guido Costamagna ${ }^{1,2}$
}

Institutions

1 Digestive Endoscopy Unit, Fondazione Policlinico Universitario A. Gemelli IRCCS, Rome, Italy

2 Centre for Endoscopic Research Therapeutics and Training (CERTT), Università Cattolica del Sacro Cuore, Rome, Italy

3 Pathology Unit, Fondazione Policlinico Universitario A. Gemelli IRCCS, Rome, Italy

4 Department of Pathology, Università Cattolica del Sacro Cuore, Rome, Italy

submitted 16.11 .2020

accepted after revision 20.1.2021

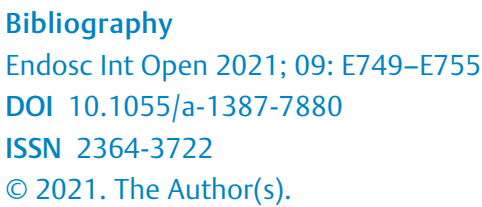

\section{ABSTRACT}

Background and study aims Intraductal extension of ampullary adenoma represents a challenging endoscopic issue. Intraductal radiofrequency ablation (RFA) has been recently suggested, but evidence and standardization of this technique are still lacking. This study aimed to provide a long-term evaluation of clinical efficacy and safety of intraductal RFA ablation with a standardized algorithm of treatment.

Patients and methods Data were prospectively collected from consecutive patients with intraductal extension of adenomatous ampullary lesions from January 2016 to November 2018. Endpoints of the study were clinical success evaluated on histology results at the last follow-up, technical success, and adverse events assessment.

Results Nine patients with intraductal (biliary \pm pancreatic) extension of ampullary adenomas were treated with RFA during the study period. Histology on the papillectomy specimen confirmed intraductal involvement with lowgrade dysplasia (LGD) in five cases (56\%), high-grade dysplasia (HGD) in three (33\%), and HGD with intramucosal adenocarcinoma in one patient (11\%). Additional argon plasma coagulation to ablate the adenoma on the duodenal mucosa was applied in five patients (56\%). Technical success was $100 \%$. One patient $(11 \%)$ with failed pancreatic stenting, developing acute pancreatitis after RFA, recovered with medical therapy. After a median follow-up of 21 months (IQR 20-31), six patients (67\%) achieved clinical success being free of recurrence, whereas one was diagnosed with persistence of adenocarcinoma, one with recurrent $\mathrm{HGD}$, and one with recurrent LGD.

Conclusions In our experience, intraductal RFA achieved acceptable results after a 2-year follow-up. Further studies are required to confirm our results and to select those patients most likely to respond.

\section{Introduction}

Endoscopic snare papillectomy (ESP) is a well-established endoscopic technique for the management of benign tumors of the papilla of Vater with a reported complete resection rate of $67 \%$ to $92 \%$ [1-5]. The ability of ESP in obtaining a complete resection is decreased in case of intraductal extension of the ampullary lesion [6]. Traditionally, surgical intervention (pancreatico- 
duodenectomy and transduodenal ampullectomy) has been considered as the mainstay of treatment for intraductal ampulloma $[1,7]$, even though burdened by a relevant morbidity and mortality rate (1-9\%) [1,8-10]. Of note, some authors have recently suggested resection of the intraductal tissue after its exposure with an extraction balloon $[11,12]$ but this technique may be technically challenging [13].

Recent studies have reported that endoscopic intraductal radio frequency ablation (RFA) can represent a minimally invasive approach to achieve a radical treatment of residual neoplastic tissue extending from the papilla into the common bile duct (CBD) and/or the pancreatic duct (PD) after ESP by inducing coagulative necrosis of the target tissue [14-19]. However, only scant data are available, and the current role of intraductal RFA as well as the modality of ablation still needs to be clarified and standardized.

The aim of this study was to provide a long-term evaluation of clinical efficacy and safety of intraductal RFA ablation to treat CBD and/or PD extension of ampullary adenomas, and to suggest a standardized algorithm and protocol of ablation.

\section{Patients and methods}

From January 2016 to November 2018 consecutive patients with intraductal extension of adenomatous lesions of the papilla of Vater were prospectively recorded. The Ethics Committee of the Catholic University of Rome approved data collection and follow-up of patients undergoing endoscopic therapy for ampullary lesions $(0040189 / 16)$.

The primary endpoint of the study was the evaluation of RFA clinical success defined as histologic absence of recurrence based on extensive follow-up biopsies from the site of papillectomy and distal bile duct, as previously reported [16]; secondary endpoints were evaluation of technical success defined as the ability to deliver RFA on the targeted areas and assessment of adverse events (AEs).

The following data were recorded: sex, age, clinical presentation, ampullary lesion size of histological specimen, length of intrabiliary and/or intrapancreatic growth (measured on X-ray images after adjusting to the endoscope diameter as a reference), number of RFA procedures, procedure-related adverse events (AEs), need for concomitant duodenal ablation by Argon Plasma Coagulation (APC), and intraductal biopsy results during follow-up.

Exclusion criteria were ampullary adenoma $>4 \mathrm{~cm}$ and jaundice at diagnosis of the ampullary lesion.

\section{Medical work-up and endoscopic intraductal RFA ablation}

All patients with histologic confirmation of ampullary adenoma underwent endoscopic evaluation of the papilla with a sideviewing endoscope. Preoperative EUS and/or computed tomography scan and/or magnetic resonance imaging were performed only in patients with large lesions $(>2 \mathrm{~cm})$, with features of malignancy (ulcerations, indurations, altered mucosal pattern), or those with severe cholestasis to evaluate the intraductal and transmural extension and to detect regional or distant

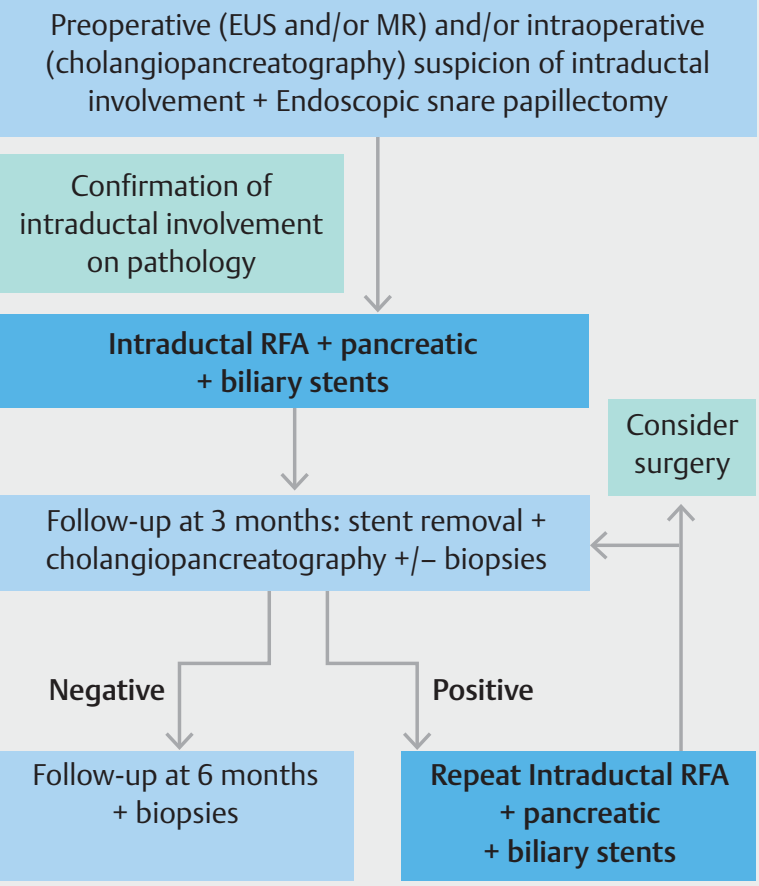

- Fig. 1 Flowchart of management and follow-up in the case of ampullary adenoma with intraductal extension at our Institute.

metastases ( $\mathbf{F i g . 1}$ ). Patients with superficial ampullary lesions underwent ESP without submucosal injection and a standard braided, oval polypectomy snare was used aiming at en-bloc resection, followed by prophylactic pancreatic stenting. In case of large ampullary adenomas laterally spreading on the duodenal wall, submucosal injection with piecemeal resection was planned.

Before ESP, cholangiogram and pancreatogram were performed to assess the presence and the length of the intraductal extension. When the intraductal neoplastic tissue was confirmed on final histology, intraductal RFA ablation was proposed to the patient after careful information concerning the lack of long-term evidence of the results. Intraductal RFA was performed at least 1 month after endoscopic papillectomy to obtain results of final histology and to avoid possible thermal injury over the resected area.

All RFA ablation procedures were performed with therapeutic duodenoscopes (Olympus TJF series, Japan) under general anesthesia with orotracheal intubation. Preoperative rectal indomethacin was administered to all patients. Intraductal RFA was delivered using the VIVA Combo generator (STARmed, Seoul, South Korea), which provides a precise power setting, target temperature, and impedance control (sudden changes of temperature, and impedance during RFA application automatically shut down the energy for safety). RFA was applied as follows: $10 \mathrm{~W}, 80^{\circ}$, and 120 -second duration in the biliary and pancreatic duct. Dedicated 7 Fr diameter, 18-mm or 33-mm probes (ELRA catheter, Seoul, South Korea) were used according to the length of the intraductal extension ( $\triangleright$ Fig.2). After 

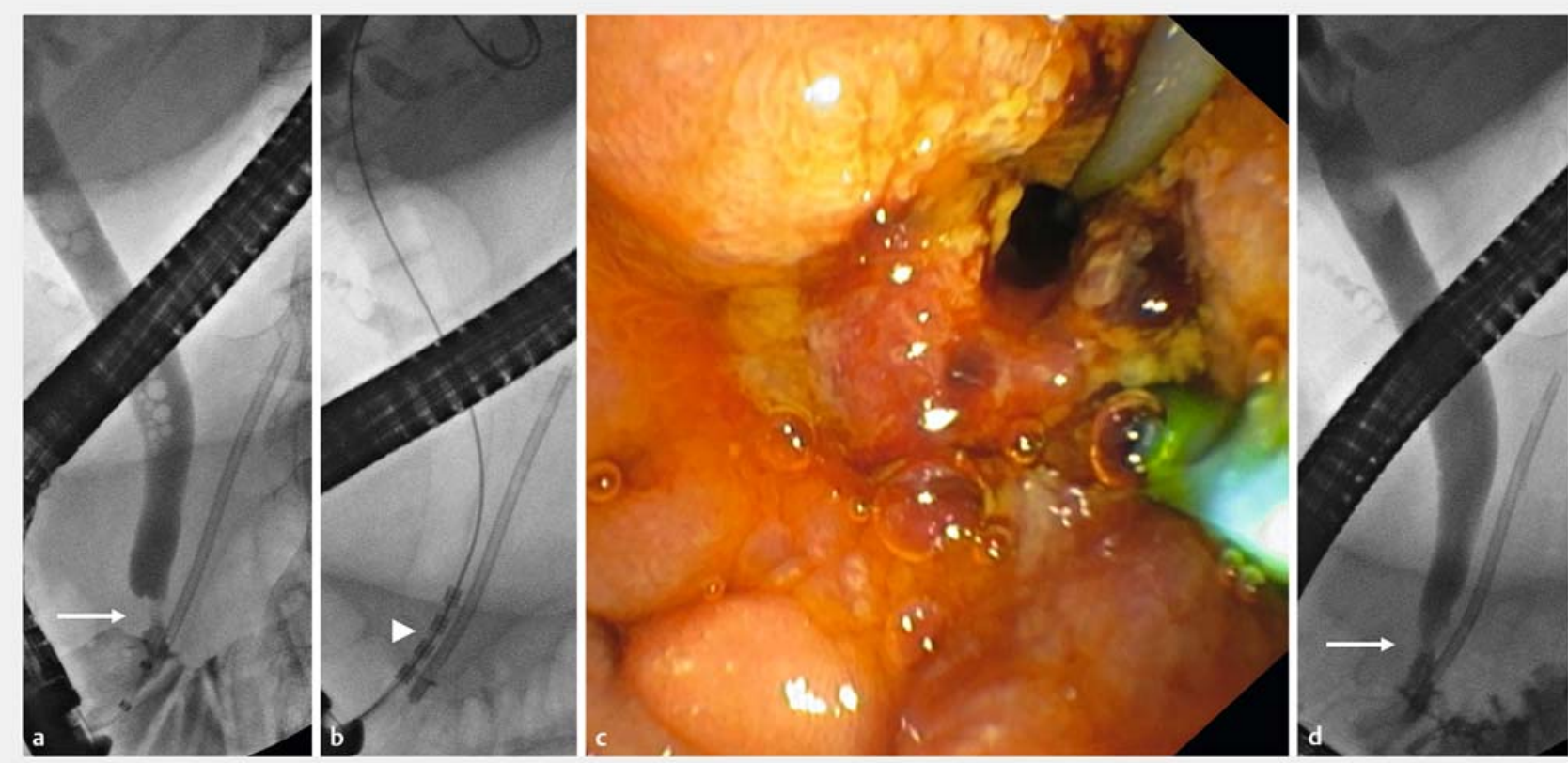

- Fig. 2 Intrabiliary radio frequency ablation (RFA). a Cholangiogram with intrabiliary ingrowth (arrow) after papillectomy and pancreatic stenting. RFA application showing $\mathbf{b}$ the intraductal probe (arrowhead), and $\mathbf{c}$ endoscopic results. $\mathbf{d}$ Resolution of the intrabiliary defect after RFA (arrow).

RFA treatment, a balloon sweep was performed to remove necrotic debris and to assess treatment response on cholangiography or pancreatography. When feasible, pancreatic and biliary plastic stents were inserted to prevent pancreatitis and post-RFA strictures of the targeted duct. The choice of the type of stent was left to the operator's preference and clinical judgment. Following the institute protocol, patients were treated with preoperative rectal non-steroidal anti-inflammatory drugs, remained with nothing-per-mouth for 12 hours, and were discharged on postoperative day 1 in the absence of AEs. Intraprocedural and early AEs (<30 days) were recorded.

\section{Follow-up}

Three months after RFA treatment, patients underwent duodenoscopy to remove biliary and pancreatic stents. At the same time, the papillary area was evaluated with Narrow Band Imaging, a cholangiogram and/or a pancreatogram were performed to check for intraductal remnant tissue, and intrabiliary biopsies under fluoroscopic control were systematically obtained following a standardized algorithm ( $>$ Fig. 1). In case of biopsy sampling still positive for adenomatous tissue, a second RFA treatment was planned. When biopsies resulted negative, patients underwent follow-up with liver function tests every 3 months for 1 year, and duodenoscopy after 3, 6, and 12 months, then annually. During follow-up, systematic biopsy sampling was carried out for the first year, then performed in the case of suspicion for local recurrence. If local recurrence was confirmed at histopathological analysis, either RFA treatment was repeated or the patient was referred to adjuvant treatments or surgery after a multidisciplinary discussion. If peri-ampullary remnant or recurrent tissue was detected, concomitant APC ablation of the external adenomatous tissue was performed during the RFA session.

\section{Statistical analysis}

Descriptive analysis was obtained reporting mean/median or percentage, wherever suitable. Statistics were performed using SPSS v. 24 (IBM SPSS Statistics for Mac, Armonk, New York, IBM Corp).

\section{Results}

During the study period, 31 patients underwent ESP; of these, nine (median age 74 [IQR 66-78] years; 89\% women) had intraductal extension of ampullary adenomatous lesions and were treated with RFA after ESP.

The clinical presentation included: anicteric cholestasis $(n=$ 4 ), incidental finding during upper GI endoscopy for dyspepsia $(n=3)$, acute cholangitis $(n=1)$ in a patient with concomitant common bile duct stone, and biliary colic $(n=1)$. Pre-ESP histology, ductal involvement and extension, histologic assessment after ESP, size of the ampullary lesion measured on histology, number of RFA treatments, biliary stenting, and follow-up are summarized in $>$ Table 1. One patient diagnosed with intramucosal adenocarcinoma after ESP was included in our study due to the refusal of surgery. One patient was considered as a poor candidate for surgery, whereas the other seven chose to undergo to endoscopic treatment after discussion with a bilio-pancreatic surgeon. 
- Table 1 Patient characteristics and outcomes.

\begin{tabular}{|c|c|c|c|c|c|c|c|c|c|c|}
\hline $\mathbf{N}$ & $\begin{array}{l}\text { Adenoma } \\
\text { size } \\
(\mathrm{mm})\end{array}$ & $\begin{array}{l}\text { Adenoma } \\
\text { pathology }\end{array}$ & $\begin{array}{l}\text { Duct in- } \\
\text { volved }\end{array}$ & $\begin{array}{l}\text { Intraduc- } \\
\text { tal exten- } \\
\text { sion } \\
(\mathrm{mm})\end{array}$ & $\begin{array}{l}\text { Intra- } \\
\text { ductal } \\
\text { pathol- } \\
\text { ogy }\end{array}$ & $\begin{array}{l}\text { RFA ses- } \\
\text { sions } \\
\text { (n) }\end{array}$ & $\begin{array}{l}\text { Concomi- } \\
\text { tant thera- } \\
\text { pies }\end{array}$ & $\begin{array}{l}\text { Biliary } \\
\text { stents } \\
\text { (n) }\end{array}$ & $\begin{array}{l}\text { Follow-up } \\
\text { from papil- } \\
\text { lectomy } \\
\text { (mo) }\end{array}$ & $\begin{array}{l}\text { Follow- } \\
\text { up from } \\
1^{\text {st }} \text { RFA } \\
\text { (mo) }\end{array}$ \\
\hline 1 & 20 & $\begin{array}{l}\text { HGD with } \\
\text { intramuco- } \\
\text { sal adeno- } \\
\text { carcinoma }\end{array}$ & CBD & 6 & $\begin{array}{l}\text { Adeno- } \\
\text { carcino- } \\
\text { ma }\end{array}$ & 1 & - & 2 & 47 & 46 \\
\hline 2 & 8 & LGD & CBD & 6 & LGD & 4 & $\begin{array}{l}\text { APC (4^ } \\
\text { session) }\end{array}$ & 2 & 44 & 42 \\
\hline 3 & $\begin{array}{l}40 \text { (ped- } \\
\text { uncula- } \\
\text { ted) }\end{array}$ & LGD & CBD & $\begin{array}{l}\text { Present } \\
\text { on histol- } \\
\text { ogy only }\end{array}$ & LGD & 1 & - & 2 & 32 & 31 \\
\hline 4 & 16 & HGD & CBD & $\begin{array}{l}\text { Present } \\
\text { on histol- } \\
\text { ogy only }\end{array}$ & LGD & 2 & $\begin{array}{l}\operatorname{APC}\left(2^{\wedge}\right. \\
\text { session })\end{array}$ & 2 & 25 & 20 \\
\hline 5 & 14 & HGD & CBD & 13 & HGD & 2 & - & 2 & 24 & 24 \\
\hline 6 & 16 & LGD & CBD & 6 & $\begin{array}{l}\text { LGD } \\
\text { (serra- } \\
\text { ted) }\end{array}$ & 1 & $\mathrm{APC}$ & 2 & 23 & 21 \\
\hline 7 & 18 & HGD & CBD & $\begin{array}{l}\text { Present } \\
\text { on histol- } \\
\text { ogy only }\end{array}$ & HGD & 1 & APC & 3 & 79 & 20 \\
\hline 8 & 25 & LGD & $\begin{array}{l}\mathrm{CBD}+ \\
\mathrm{PD}\end{array}$ & $9+9$ & LGD & 2 & APC & 3 & 20 & 19 \\
\hline 9 & 17 & HGD & CBD & 14 & HGD & 1 & - & 3 & 41 & 13 \\
\hline
\end{tabular}

All ESP were performed en-bloc. Histopathological analysis on the specimen revealed positive lateral margins in one case. On pathological assessment biliary and pancreatic margins cannot be evaluated separately because the depth of endoscopic papillectomy is limited to the ampullary margin which was positive in all the cases.

In eight of nine cases no carcinoma was detected and infiltration was therefore not present due to the adenomatous nature of the lesion. In the case in which focal carcinoma was present, this was intramucosal and limited to lamina propria.

CBD involvement on histology and/or cholangiopancreatography was reported in eight patients (89\%) for a median extension of $6 \mathrm{~mm}$ (IQR 6-11), whereas only one patient (11\%) was treated for both CBD and PD involvement. Intraductal RFA was performed on average 1.5 months (IQR 1-5) after ESP. Technical success was $100 \%$. Pancreatic plastic stenting was successful in all patients after the first session of RFA (2, $5 \mathrm{Fr}$; 6, $7 \mathrm{Fr} ; 1,10 \mathrm{Fr}$ ), but failed one patient during the second RFA procedure. Clinical success at a median follow-up of 21 months (IQR 20-31) was achieved by six patients (66.7\%) (> Table 2 ). Among clinical failures, the patient diagnosed with adenocarcinoma finally accepted surgical intervention (pancreaticoduodenectomy) for the persistence of adenocarcinoma after one session of RFA; one patient with LGD did not respond to four sessions of RFA and one session of duodenal APC but refused further treatments including surgery; one patient with preoperative HGD underwent two sessions of RFA without success but was effectively managed with external radiotherapy and follow-up biopsies were negative 18 months after radiotherapy. One session of APC was performed in five patients (56\%) during follow-up. Among AEs, one patient (11\%), where pancreatic stenting failed during the second RFA procedure, developed acute pancreatitis and recovered without sequelae after conservative management; this patient underwent other two sessions of RFA after successful pancreatic stenting without development of further complications. A mean number of two $10 \mathrm{Fr}$ (IQR 2-3) biliary plastic stents were placed after RFA and removed after a mean time of 2.5 months (IQR 2-3); no patient developed symptomatic biliary strictures during follow-up leading to cholangitis or abnormal liver function tests.

\section{Discussion}

Intrabiliary and/or intrapancreatic growth of ampullary tissue has been traditionally considered as a general contraindication for an endoscopic approach, and its optimal treatment is still a matter of debate [20-22]. 


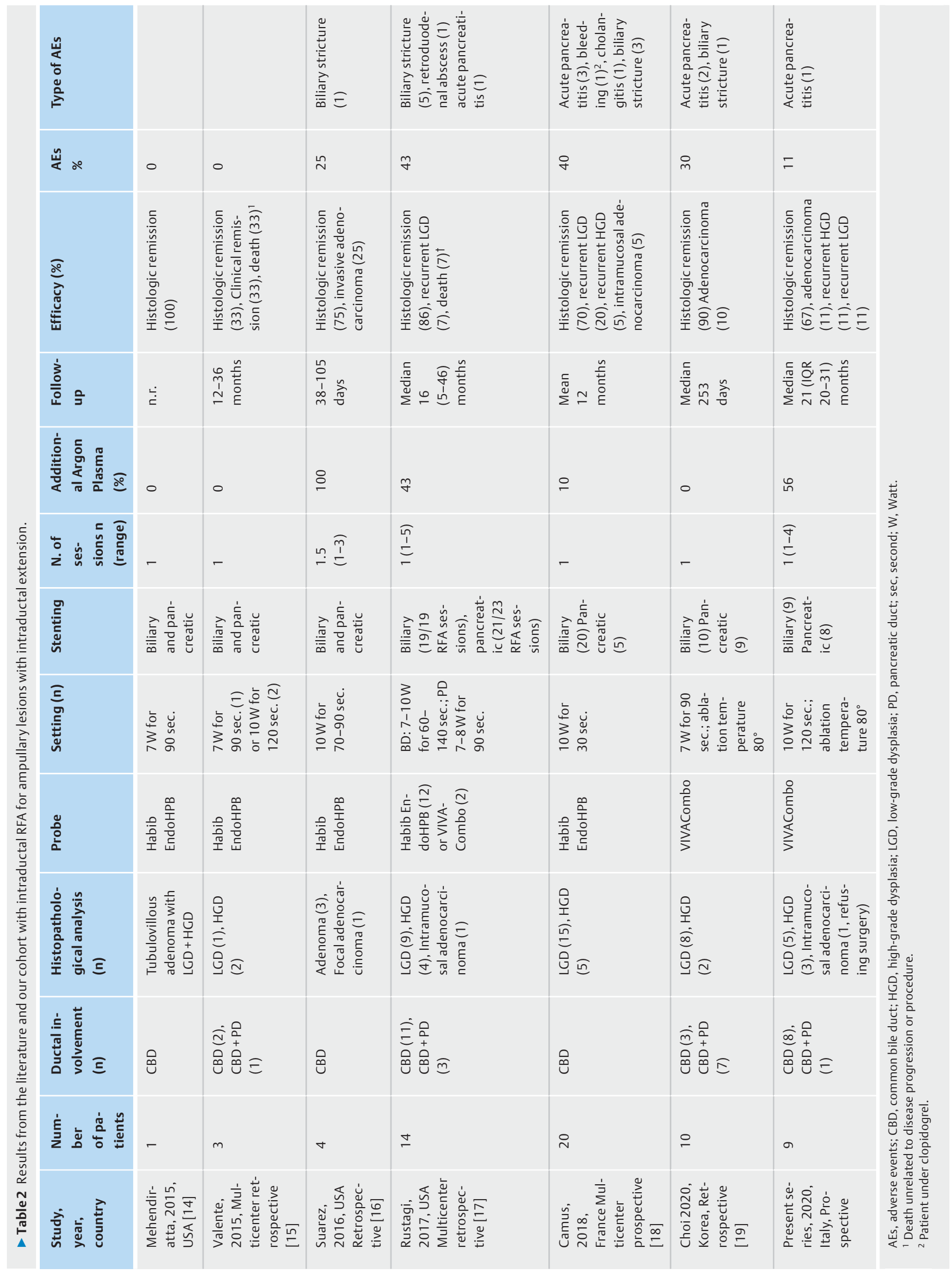


In this study, we present a series of adenomatous lesions of the papilla of Vater with intraductal extensions managed with ESP and RFA. The high incidence of intraductal extension of ampullary adenomas $(29 \%, 9 / 31)$ in our series, is possibly related to a closer attention to the intraductal extension since the availability of RFA facilities in our unit.

To provide a more meaningful comparison of intraductal RFA outcomes, the results of our study should be contextualized in light of data from other series $>$ Table 2) [14-19].

The histological result was not evaluated at long-term follow-up in most of the series $[14,16]$, and only three studies reported outcomes in more than five cases [17-19]. In their study on 20 patients, Camus et al. reported a clinical success of $70 \%$ at 12 months [18], whereas Rustagi et al. achieved histologic remission in $86 \%$ of patients [17]. In our series, clinical success was obtained in $67 \%$ of patients.

When considering ablation settings used in literature, a relevant heterogeneity in terms of time, duration, and intensity of electric current have been applied ( $>$ Table 2 ) [14-19]. In our study, we chose to perform all the procedures with the same settings ( $10 \mathrm{~W}$ for 120 seconds at a temperature of $80^{\circ}$ ) without any relevant $A E s$ except one case of pancreatitis occurring when pancreatic stenting failed. Our study has a low rate of adverse events ( $\triangleright$ Table 2 ) similar to a recent experience [19] using the same temperature-controlled generator to ablate intraductal ampullary lesions; maybe this specifically designed RFA generator (VivaCombo) is more precise and safe than the Habib probe, which is connected to a standard electrosurgical unit with limited setting possibilities [14-18].

Concerning the safety of this procedure, the main AE described in literature has been the occurrence of biliary stenosis, which has been reported by 10 of 61 patients (16\%) ( Table 2 ) [14-19]. In addition, the risk of biliary strictures was confirmed in animal models, suggesting that systematic stenting after the procedure might be useful [23]. Rustagi et al. did not perform systematic biliary stenting and reported a relatively high stricture rate (36\%) [17]. In the study by Suarez et al. one case of biliary stenosis was described [16]. In our cohort patients received always multiple biliary plastic stents and RFA-related biliary stenosis was absent at follow-up. Another option is the insertion of a fully covered self- expandable metal stent (FCSEMS), although with an increased risk of stent migration and higher costs.

Furthermore, we always tried to perform pancreatic stenting to prevent PD injury-related AEs. Post-RFA acute pancreatitis occurred in a single patient with failed pancreatic stent insertion, suggesting its importance to prevent RFA-related pancreatitis, together with pharmacological prophylaxis with rectal NSAIDs, which was systematically administered in our series. Interestingly the same patients received further and uneventful intrabiliary RFA after successful pancreatic stenting.

An alternative strategy for patients with intraductal ingrowth of papillary lesions was recently suggested, using a wire-guided cystotome for the application of a monopolar current on the intraductal tissue [13]. Curative resection was achieved in $100 \%$ (12/12) cases, with a rate of $25 \%$ of complications, including one case of pancreatitis and one of pancreatic duct stenosis [13]. Comparative studies may help choosing the most effective and safe technique in this highly specialized field.

Strengths of this study include the long follow-up reported, as well as the detailed and standardized protocol of ablation and patient management in a field that is still poorly understood.

Nevertheless, our findings need to be interpreted in light of the study limitations. First, the sample size was small. Second, due to the limited sample size and the low number of events, we could not perform a multivariate analysis assessing the predictors of patient- or disease-specific response characteristics. Third, a control group was absent (i. e. photodynamic therapy).

\section{Conclusions}

Future studies can consider our standardized protocols to focus on the identification of patients most likely to respond to intraductal RFA, supporting more individualized therapy. Surveillance after intraductal RFA should be assessed, also investigating the role of EUS in this setting. Further randomized studies are required to assess the real role of this strategy in the endobiliary space.

\section{Acknowledgments}

The authors thank Franziska Lohmeyer for her English language assistance.

\section{Competing interests}

Dr. Costamagna is on the Advisory Boards of Cook Medical, Olympus, and Ethicon and has received Research Grants from Boston Scientific Corp. and Apollo Endosurgery.

References

[1] Chathadi KV, Khashab MA. ASGE Standards of Practice Committee. et al. The role of endoscopy in ampullary and duodenal adenomas. Gastrointest Endosc 2015; 82: 773-781

[2] Kang SH, Kim KH, Kim TN et al. Therapeutic outcomes of endoscopic papillectomy for ampullary neoplasms: retrospective analysis of a multicenter study. BMC Gastroenterol 2017; 17: 69

[3] Binmoeller KF, Boaventura S, Ramsperger K et al. Endoscopic snare excision of benign adenomas of the papilla of Vater. Gastrointest Endosc 1993; 39: 127-131

[4] Vogt M, Jakobs R, Benz C et al. Endoscopic therapy of adenomas of the papilla of Vater. A retrospective analysis with long-term follow-up. Dig Liver Dis 2000; 32: 339-345

[5] Haraldsson E, Swahn F, Verbeke C et al. Endoscopic papillectomy and KRAS expression in the treatment of adenoma in the major duodenal papilla. Scand J Gastroenterol 2015; 50: 1419-1427

[6] Bohnacker S, Seitz U, Nguyen D et al. Endoscopic resection of benign tumors of the duodenal papilla without and with intraductal growth. Gastrointest Endosc 2005; 62: 551-560

[7] Ceppa EP, Burbridge RA, Rialon KL et al. Endoscopic versus surgical ampullectomy: an algorithm to treat disease of the ampulla of Vater. Ann Surg 2013; 257: 315-322 
[8] Cahen DL, Fockens P, de Wit LT et al. Local resection or pancreaticoduodenectomy for villous adenoma of the ampulla of Vater diagnosed before operation. Brit J Surg 1997; 84: 948-951

[9] Jordan PH Jr, Ayala G, Rosenberg WR et al. Treatment of ampullary villous adenomas thatmay harbor carcinoma. J Gastrointest Surg 2002; 6: 770-775

[10] Tran TC, Vitale GC. Ampullary tumors: endoscopic versus operative management. Surg Innov 2004; 11: 255-263

[11] Kim JH, Moon JH, Choi HJ et al. Endoscopic snare papillectomy by using a balloon catheter for an unexposed ampullary adenoma with intraductal extension (with videos). Gastrointest Endosc 2009; 69: 1404-1406

[12] Aiura K, Imaeda H, Kitajima M et al. Balloon-catheter-assisted endoscopic snare papillectomy for benign tumors of the major duodenal papilla. Gastrointest Endosc 2003; 57: 743-747

[13] Pérez-Cuadrado-Robles E, Piessevaux H, Moreels TG et al. Combined excision and ablation of ampullary tumors with biliary or pancreatic intraductal extension is effective even in malignant neoplasms. United European Gastroenterol J 2019; 7: 369-376

[14] Mehendiratta V, Desilets DJ. Use of radiofrequency ablation probe for eradication of residual adenoma after ampullectomy. Gastrointest Endosc 2015; 81: 1055-1056

[15] Valente R, Urban O, Del Chiaro M et al. ERCP-directed radiofrequency ablation of ampullary adenomas: a knife-sparing alternative in patients unfit for surgery. Endoscopy 2015; 47: E515-E516
[16] Suarez AL, Coté GA, Elmunzer B]. Adjunctive radiofrequency ablation for the endoscopic treatment of ampullary lesions with intraductal extension (with video). Endosc Int Open 2016; 4: E748-E751

[17] Rustagi T, Irani S, Reddy DN et al. Radiofrequency ablation for intraductal extension of ampullary neoplasms. Gastrointest Endosc 2017; 86: $170-176$

[18] Camus M, Napoléon B, Vienne A et al. Efficacy and safety of endobiliary radiofrequency ablation for the eradication of residual neoplasia after endoscopic papillectomy: a multicenter prospective study. Gastrointest Endosc 2018; 88: 511-518

[19] Choi YH, Yoon SB, Chang JH et al. The safety of radiofrequency ablation using a novel temperature-controlled probe for the treatment of residual intraductal lesions after endoscopic papillectomy. Gut Liver 2020: Jul 6. Epub ahead of print. doi:10.5009/gnl20043

[20] Desilets DJ, Dy RM, Ku PM et al. Endoscopic management of tumors of the major duodenal papilla: refined techniques to improve outcome and avoid complications. Gastrointest Endosc 2001; 54: 202-208

[21] Catalano MF, Linder JD, Chak A et al. Endoscopic management of adenoma of the major duodenal papilla. Gastrointest Endosc 2004; 59: 225-232

[22] Norton ID, Gostout C], Baron TH et al. Safety and outcome of endoscopic snare excision of the major duodenal papilla. Gastrointest Endosc 2002; 56: 239-243

[23] Cho JH, Jeong S, Kim EJ et al. Long-term results of temperature-controlled endobiliary radiofrequency ablation in a normal swine model. Gastrointest Endosc 2018; 87: 1147-1150 